\section{Poster 146 \\ The Reliability of the Clinical Diagnosis in Persons Offered Surgery for Lumbar Spinal Stenosis.}

Andrew J. Haig, MD (University of Michigan, Ann Arbor, MI); Peter Henke, MD; Sierra Loar, MPA; Paul Park, MD; Christy Tomkins, PhD; Juan Valdivia; Karen S. Yamakawa, MS

Disclosures: A. J. Haig, Rehabilitation Team Assessments, LLC, Ownership or partnership; The International Rehabilitation Forum, Non-remunerative positions of influence; The University of Michigan, Employment.

Objective: To determine if expert clinical history and physical examination can differentiate persons offered surgery for spinal stenosis, persons with vascular claudication, and asymptomatic age-matched controls.

Design: IRB approved, NIH-funded, prospective, controlled, masked diagnostic trial.

Setting: Academic medical center.

Participants: Patients with no polyneuropathy risk, negative ankle brachial index (ABI) and no previous surgery who were offered surgery for lumbar stenosis; persons diagnosed with vascular claudication, positive $\mathrm{ABI}$, and no back pain; and asymptomatic volunteers. Patients were found by systematic review of vascular and spine clinic records, control subjects by community recruitment.

Interventions: Examination and diagnosis by faculty vascular and neurosurgeons, masked to imaging and vascular tests, who reviewed extensive questionnaires, performed a comprehensive and open-ended spine and vascular history and physical examination.

Main Outcome Measures: Agreement between examiners.

Results: Among 234 persons preliminarily screened, 79 qualified and 48 completed all components, including 15 (60\%) of surgical stenosis, $6(50 \%)$ of vascular claudication, and $27(64 \%)$ of asymptomatic recruits. While there was good agreement (Kappa 0.637) between them, the surgeons did disagree on $20 \%$ of diagnoses. The neurosurgeons classified 4 (24\%) of 17 persons who had been offered spine surgery as asymptomatic and 1 as vascular.

Conclusions: Spinal stenosis has been defined as a clinical entity associated with a radiological finding. Recent studies have exposed the lack of a concrete radiological criteria. Now this study of clinical diagnosis, in the ideal situation of persons stringently screened and extensively examined by experienced specialists, found disagreement about diagnosis in $1 / 5$ of persons who were offered surgical intervention for the syndrome. This lack of reliable clinical and radiological criterion standards may explain some of the arbitrary and exponential rise in stenosis surgery. A specific clinical criterion for the clinical syndrome of lumbar spinal stenosis may make the diagnosis less ambiguous.

\section{Poster 148 \\ The Thirteen-Point Shoulder Ultrasound Examination. \\ Don Buford, MD (Sports Medicine Clinic of North Texas, Dallas, TX); Ben DuBois, MD.}

Disclosures: D. Buford, Conmed Linvatec, Consulting fees or other remuneration; Orthotalk, Inc, Consulting fees or other remuneration.

Discussion: With the continued rise in the cost of health care in the United States, all involved in health care should be sensitive to the cost of health care delivery. Within musculoskeletal specialties, one reason for the rising cost of health care is the rising cost in diagnosing patients with musculoskeletal pathology. Often, the clinician needs additional information beyond the history, physical examination and routine radiographs and opts to obtain a magnetic resonance imaging (MRI) scan or a computed tomography (CT) scan for additional diagnostic information. These tests are relatively expensive and may expose the patient to additional radiation. It is our opinion that for certain musculoskeletal conditions around the shoulder, office-based ultrasound is a modality that has a significantly lower cost, is more efficient, and provides a radiation free means of obtaining diagnostic information. Current commercially available portable ultrasound machines can provide for expanded high-resolution images of the soft tissue, extra-articular anatomy around the shoulder. In this paper, we describe a new, standardized 13-point shoulder ultrasound examination to provide a simplified template for musculoskeletal clinicians. We have specifically tailored our examination to be efficient and comfortable for both surgical and post-surgical patients. We also provide positioning tips and image orientation guidelines designed to shorten the learning curve for clinicians with experience evaluating radiographs, MRI studies, and CT studies.

\section{Poster 149}

\section{Therapeutic Outcomes of Conservative Treatment Package for Surgical Candidates With Lumbar Disk Herniation (2 year).} Youngkwon Choi, MD (Jaseng Medical Foundation, Seoul, Republic of Korea); JoonShik Shin; Yousuk Youn, Head.

Disclosures: Y. Choi, None.

Objective: The purpose of this prospective observational cohort study was to prove the effectiveness of the integrative treatment package for surgical candidates with disk herniation.

Design: Prospective observational study (2-year follow-up). Setting: An outpatient clinic in Seoul, Korea.

Participants: We selected 92 consecutive low back pain patients (20 to 60 years old) with lateral radicular pain to lower extremities diagnosed as lumbar disk herniation (prolapse extrusion) confirmed by MRI since November 